\title{
Heartbeat: public involvement in cardiovascular research
}

doi:10.1136/heartjnl-2021-319452

Current clinical cardiovascular disease (CVD) research primarily is 'about' patients with CVD but performed by academic researchers with little or no input from the public or patients. As concisely summarised in the Cardiology in Focus article ${ }^{1}$ in this issue of Heart, public involvement in research both empowers patients and the public and can point researchers towards critical questions or outcomes important to patients. Public involvement in cardiovascular research also has the potential to reduce global inequity in health research. Even so, it can be daunting for a clinical investigator to actively involve patients, other than as enrolled participants, due to lack of knowledge about why or how to do so. The article by Ramakrishnan and Miller provides practical advice, with links to more detailed information online, that will help researchers effectively involve the public in clinical cardiovascular research; an approach that increasingly is considered an essential element of an ethical and rigorous research study (figure 1).

Among adults with congenital heart disease (ACHD), heart failure (HF) is a primary cause of hospitalisation and mortality. In order to better define the healthcare burden of HF in ACHD patients, Burstein and colleagues ${ }^{2}$ used data from the US Nationwide Emergency Department Sample and the Nationwide Inpatient Sample which included over 31 million emergency department visits for HF. Although only $0.25 \%$ (76 557) had an underlying ACHD diagnosis, patients with ACHD were younger, had higher admission rates, a longer lengthof-stay and greater hospital costs. Importantly, mortality was higher in HF patients with ACHD, both in those with single ventricle $(6.6 \%$; OR $1.6,95 \%$ CI 1.1 to $2.3)$ or two-ventricle physiology $(6.3 \%$; OR $1.4,95 \%$ CI 1.3 to 1.5$)$ compared with patients with HF but without ACHD (5.5\%). Over the decade studied (2006-2016), ACHD hospitalisation for HF increased more than for non-ACHD patients (figure 2), yet a lower proportion

Division of Cardiology, University of Washington, Seattle, Washington, USA

Correspondence to Professor Catherine M Otto, Division of Cardiology, University of Washington, Seattle, WA 98195, USA; cmotto@uw.edu

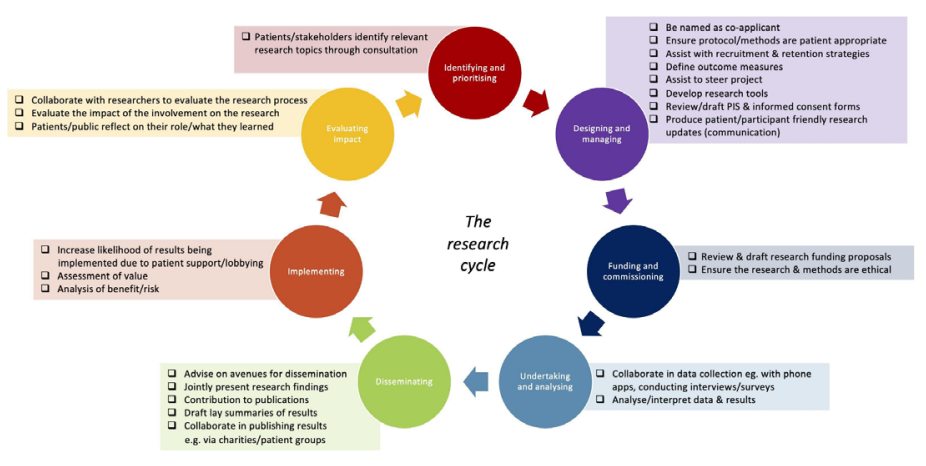

Figure 1 Ways that people can be involved in the research cycle (adapted from UK Standards for Public Involvement; https://www.invo.org.uk/).

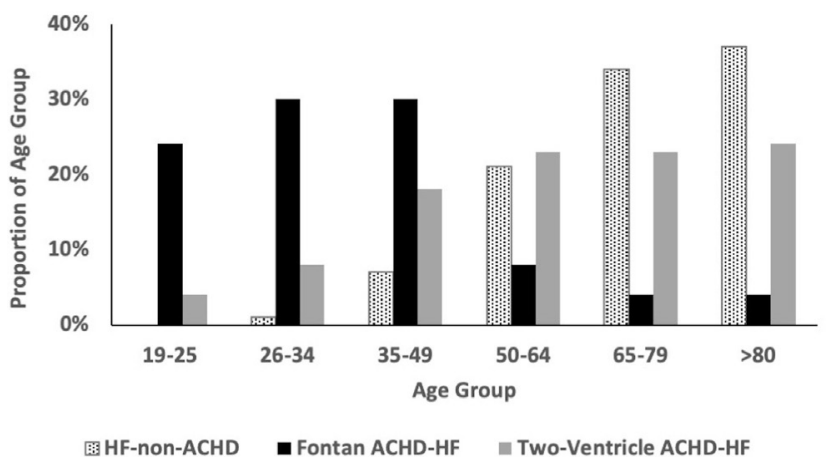

Figure 2 Age at presentation for ACHD-HF versus HF-non-ACHD visits. ACHD-HF, adults with congenital heart disease and heart failure; HF-non-ACHD, non-ACHD with HF.

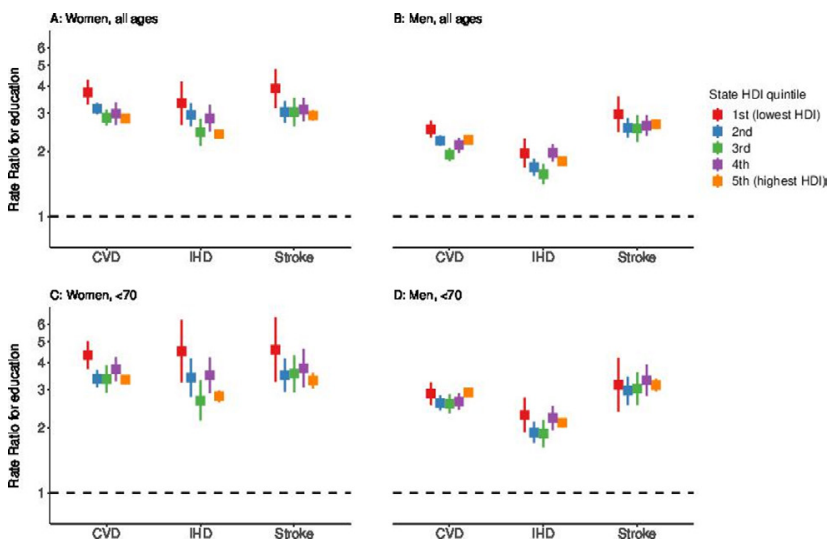

Figure 3 Rate ratios for association between education ( $<8$ vs $8+$ years) and cardiovascular mortality by quintile of state Human Development Index (HDI) in Brazil, 2010. CVD, cardiovascular disease; IHD, ischaemic heart disease.

of ACHD-HF patients received a ventricular assist device or heart transplantation.

In an editorial, Ladouceur ${ }^{3}$ comments 'The report by Burstein et al' contextualises the magnitude of the burden of ACHD-HF. It highlights interesting new issues about the utilisation of healthcare resources to manage this severe complication. Although these data will be useful, the development of cohorts of ACHD-HF is mandatory to allow advance research notably to improve ACHD-HF treatments'. 


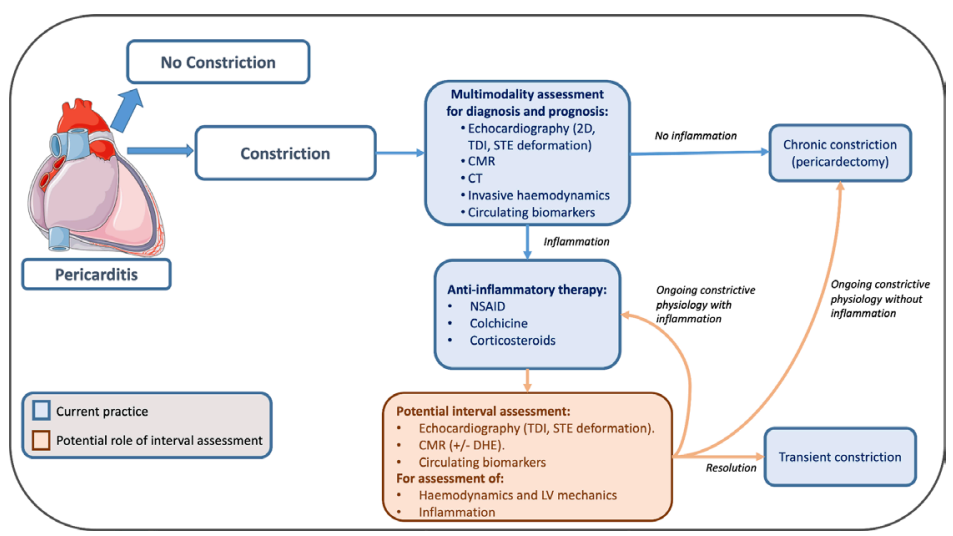

Figure 4 Potential role of interval imaging in transient pericarditis. The current clinical role of multimodality imaging in constrictive pericarditis is outlined in blue. More research is needed to support the potential role for multimodality imaging in predicting response to anti-inflammatory therapy as well as following up response to treatment, outlined in orange box. Image contains material licensed under CC-BY 3.0 (https://smart.servier.com/). 2D, two-dimensional; CMR, cardiac MRI; DHE, delayed hyperenhancement; LV, left ventricular; NSAID, non-steroidal anti-inflammatory drug; STE, speckle tracking echocardiography; TDI, tissue Doppler imaging.

\section{Asymptomatic, isolated severe aortic stenosis}

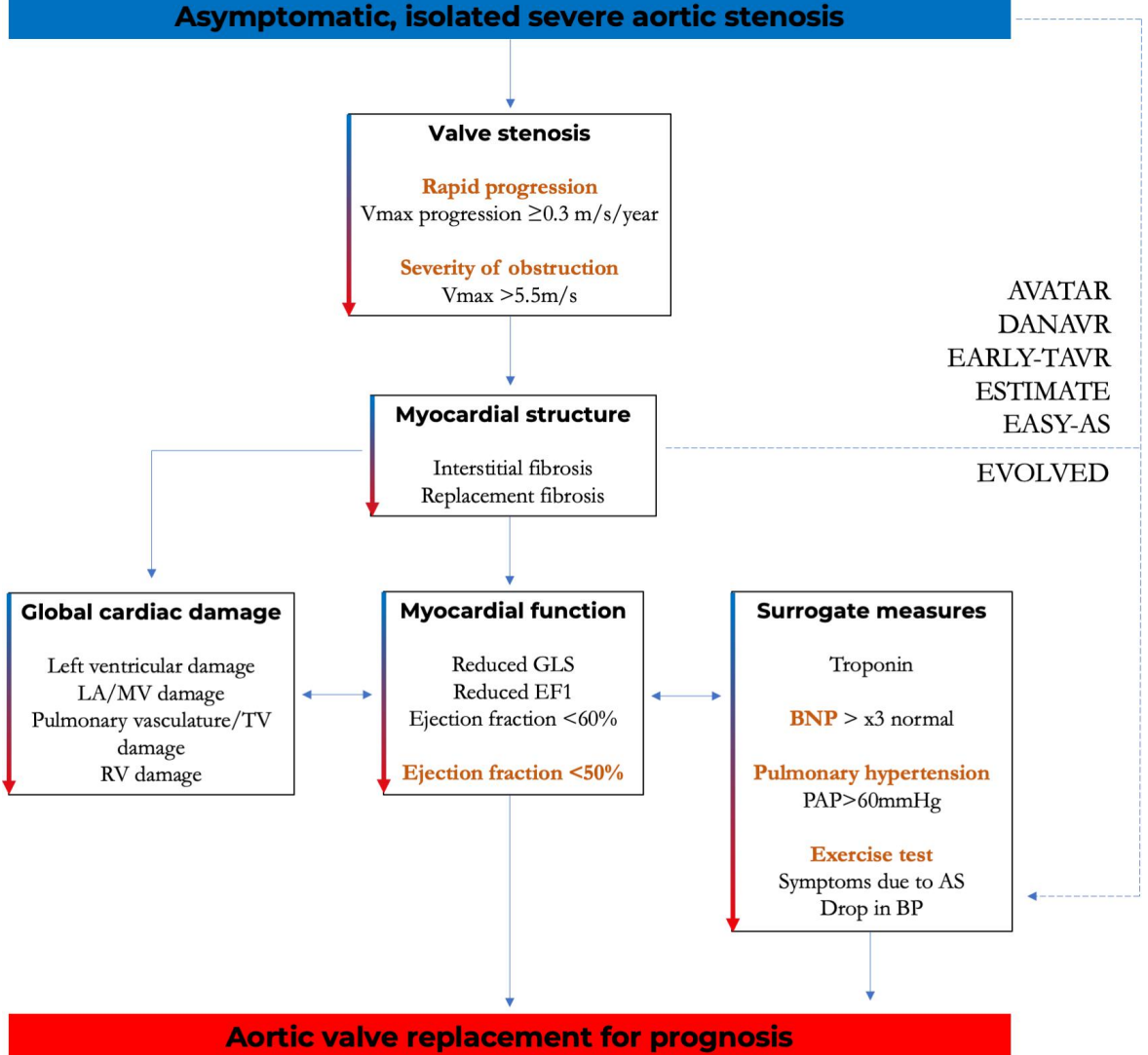

Figure 5 Conceptual flow chart of assessments in asymptomatic severe aortic stenosis. Although the disease is a spectrum and interactions are complex, it can be helpful to compartmentalise the processes that can be quantified with various assessments. Ejection fraction $<50 \%$ and exerciseinduced symptoms clearly attributable to aortic stenosis are the only two class I recommendations for aortic valve replacement in current guidelines (level of evidence C). ${ }^{1}$ Other parameters with weaker recommendations are also highlighted in orange text. To the right are the acronyms for currently enrolling randomised controlled trials of early intervention (see article for details). AS, aortic stenosis; BP, blood pressure; BNP, B-type natriuretic peptide; EF1, first-phase ejection fraction; GLS, global longitudinal strain; $L A$, left atrial; MV, mitral valve; PAP, pulmonary artery pressure; TV, tricuspid valve.
Although it has been suggested that economic development of low-and-middleincome countries might result in worsening CVD risk in higher, compared with lower, socioeconomic (SES) groups, convincing data to support this view is lacking. In this issue of Heart, Mallinson and colleagues ${ }^{4}$ used national census and mortality data for Brazil to address this issue. Age-adjusted mortality rates for both women and men with $<8$ years of education (compared with 8 or more years) was higher in the least, compared with the most, developed states with a similar pattern seen for subtypes of CVD (figure 3). The authors conclude that: 'The belief in a 'social crossover' in CVD mortality on economic development, from greater risk in high socioeconomic groups to greater risk in low socioeconomic groups, is not supported by evidence from low and middle income countries (LIMCs) .'

In the accompanying editorial, Mullachery and Bilal ${ }^{5}$ agree and conclude that this study 'challenges the validity and the generalisability of the social crossover of CVD mortality, which was first described in a specific historical context in high-income countries. Inequalities in CVD mortality and CVD risk factors in Brazil demonstrate that low SES groups are indeed at a greater risk compared with high SES groups. CVD prevention efforts should focus on low-SES populations, that continue to bear the burden of higher CVD rates.'

An interesting small study of 35 patients with transient or persistent constrictive pericarditis showed an association between use of anti-inflammatory therapy and resolution of symptoms, further supported by improvement in the echocardiographic ratio of left ventricular lateral to septal wall longitudinal strain. ${ }^{6}$ Moharram et $\mathrm{al}^{7}$ suggest it may be possible to integrate these new markers of disease activity in our approach to diagnosis and medical therapy for transient constrictive pericarditis (figure 4). In this algorithm, surgical pericardiectomy is reserved for patients with no signs of inflammation but with persistent or ongoing symptomatic constrictive pericarditis. However, additional clinical studies are needed; 'The concept of transient constrictive pericarditis is relatively new and our understanding of the underlying pathophysiological mechanisms is evolving. Using anti-inflammatory therapy without specific predictors of which patients would benefit leaves many patients with an unfavourable risk to benefit ratio.'

The Education in Heart article ${ }^{8}$ in this issue reviews the subclinical pathology that precedes symptom onset 
in adults with calcific aortic stenosis and summarises the evidence base for the ongoing trials of earlier intervention in adults with asymptomatic severe aortic stenosis (figure 5).

Funding The authors have not declared a specific grant for this research from any funding agency in the public, commercial or not-for-profit sectors.

Competing interests None declared.

Patient and public involvement Patients and/or the public were not involved in the design, or conduct, or reporting, or dissemination plans of this research.

Patient consent for publication Not required.

Provenance and peer review Commissioned; internally peer reviewed.
(C) Author(s) (or their employer(s)) 2021. No commercial re-use. See rights and permissions. Published by BMJ.

\section{(A) Check for updates}

To cite Otto CM. Heart 2021;107:771-773.

Heart 2021:107:771-773.

doi:10.1136/heartjnl-2021-319452

\section{ORCID iD}

Catherine M Otto http://orcid.org/0000-0002-05279392

\section{REFERENCES}

1 Ramakrishnan A, Miller M. A brief guide to public involvement in cardiovascular research. Heart 2021;107:851-3.
2 Burstein DS, Rossano JW, Griffis H. Greater admissions, mortality and cost of heart failure in adults with congenital heart disease. Heart 2021;107:807-13.

3 Ladouceur M. Heart failure in adults with congenital heart disease: a call for action. Heart 2021;107:774-5.

4 Mallinson PAC, Luhar S, Williamson E, et al. Socioeconomic position and cardiovascular mortality in 63 million adults from Brazil. Heart 2021:107:822-7.

5 Mullachery PH, Bilal U. Social cross-over in cardiovascular disease: context matters. Heart 2021;107:778-80

6 Sato K, Ayache A, Kumar A, et al. Improvement in left ventricular mechanics following medical treatment of constrictive pericarditis. Heart 2021;107:828-35.

7 Moharram M, Whalley G, Coffey S. Interval imaging to guide treatment in constrictive pericarditis. Heart 2021:107:781-2.

8 Bing R, Dweck MR. Management of asymptomatic severe aortic stenosis: check or all in? Heart 2021;107:842-50. 